\title{
International Comparison
}

\section{International comparison of the illuminance responsivity scales and units of luminous flux maintained at the HUT (Finland) and the NIST (USA)}

\author{
J. Hovila, P. Toivanen, E. Ikonen and Y. Ohno
}

\begin{abstract}
An international comparison has been conducted to compare the illuminance responsivity scales (A/lx) and the units of luminous flux $(\mathrm{lm})$ maintained at the National Institute of Standards and Technology (NIST, USA) and the Helsinki University of Technology (HUT, Finland). Both laboratories realize the illuminance unit by absolutely calibrated photometers and the luminous flux unit by the absolute integrating-sphere method. Standard photometers were used as transfer standards for the illuminance responsivity comparison, and standard lamps in the luminous flux comparison. The ratio of the measured illuminance responsivity values (HUT/NIST) was 0.9992 with an expanded uncertainty $(k=2)$ of 0.0013 , and the ratio of the measured luminous flux values was 1.0006 with an expanded uncertainty $(k=2)$ of 0.0018 . The relative expanded uncertainties of the agreement of the units, including the uncertainties of the realizations of the units as well as the uncertainty of the comparison, were 0.0047 and 0.0101 , respectively.
\end{abstract}

\section{Introduction}

Absolutely calibrated photometers are now commonly used in realizations of the photometric units. The use of such reference photometers leads to lower uncertainty levels and simplified measurement facilities. The trend in many national standards laboratories is therefore to develop detector-based realizations of all applicable units in photometry and radiometry. Both the HUT and the NIST base their realizations of the units of luminous intensity and illuminance on absolute responsivity calibrations of photometers [1-3].

The absolute integrating-sphere method for the realization of the unit of luminous flux ( $\mathrm{lm}$ ) was developed at the NIST [4]. Recently, this method has been implemented with small modifications at the HUT [5]. After thorough characterizations and uncertainty calculations, the HUT measurement facility was ready for the first international comparison. Although the main objective of this work was to evaluate the performance of the HUT luminous flux calibration facility, the

J. Hovila, P. Toivanen and E. Ikonen: Metrology Research Institute, Helsinki University of Technology (HUT), PO Box 3000, FIN-02015 HUT, Finland.

Y. Ohno: Optical Technology Division, National Institute of Standards and Technology (NIST), Gaithersburg, MD 20899 USA. illuminance responsivity scales $(\mathrm{A} / \mathrm{lx})$ as well as the luminous flux units were compared in order to evaluate the process in the absolute integrating-sphere method alone by determining the difference in the illuminance units. The comparison measurements were conducted at the HUT and the NIST from June to August 2000. This paper presents brief descriptions of the realizations of the units at both laboratories together with the results of the comparison.

\section{Realization and maintenance of the photometric units}

\subsection{The NIST}

The NIST illuminance unit has been realized annually based on the absolute cryogenic radiometer since 1992. It is realized and maintained via a group of eight standard photometers calibrated for illuminance responsivity (A/lx). The NIST standard photometers are composed of a silicon photodiode, a $V(\lambda)$ correction filter and a high-precision aperture, and are also equipped with a temperature sensor that allows for a correction for change in photometer temperature. The absolute spectral responsivity (A/W) of the photometers is calibrated annually by the NIST Spectral Response Calibration Facility [6], traceable to 
the NIST absolute cryogenic radiometer [7]. With the calibrated aperture area and a correction for spatial non-uniformity of response over the aperture, the illuminance responsivity (A/lx) of each photometer for $2856 \mathrm{~K}$ Planckian radiation is determined. The relative expanded uncertainty $(k=2)$ of the realization of the NIST illuminance unit is $0.39 \%$ [1]. Further details of the latest NIST photometric measurement facility and realization process are given in [8]. The illuminance responsivity measurements in this comparison were based on the NIST illuminance unit realized in 1999.

The NIST luminous flux unit has been realized from the NIST illuminance unit using the absolute integrating-sphere method since 1995 [4, 8]. A $2.5 \mathrm{~m}$ integrating sphere is now used not only for the realization of the unit but also for absolute measurement of test lamps [9]. The unit is maintained by the integrating-sphere system itself and also by a group of luminous flux reference lamps. The relative expanded uncertainty $(k=2)$ of the current NIST luminous flux unit is $0.50 \%$ [9].

While the NIST luminous flux unit is expected to be unchanged since 1995, the luminous flux measurements in this comparison were made directly against the six reference lamps that were used for the 1997 key comparison CCPR-K4 [10], in order to relate the results of this comparison directly to that of the key comparison. The relative expanded uncertainty $(k=2)$ of the 1997 NIST luminous flux unit maintained on these lamps was $0.62 \%$.

\subsection{The HUT}

The realization of the illuminance unit at the HUT is based on an absolutely characterized reference photometer utilizing a trap detector, a temperaturecontrolled $V(\lambda)$-correction filter and a high-precision aperture. The absolute responsivity of the trap detector is calibrated by a cryogenic radiometer at several laser wavelengths. The relative spectral responsivity of the trap detector is obtained by a physical model for the external quantum efficiency of the photodiodes. The spectral transmittance of the $V(\lambda)$ filter is measured using a reference spectrophotometer. The area of the aperture is measured by the direct optical method [11]. The illuminance responsivity of the reference photometer is obtained from the individual characteristics of the components, as interreflections are reduced by the use of the trap detector in the photometer. The relative expanded uncertainty $(k=2)$ of the realization of the illuminance unit is $0.18 \%$.

The reference photometer is characterized once a year. Between the realizations, the unit is maintained by the reference photometer, a group of standard lamps, and by two commercial photometers (PRC TH15-BA0-D8). A more detailed description of the realization and maintenance of the unit is presented in [3].
The luminous flux unit at the HUT is realized by the absolute integrating-sphere method with a slightly different measurement set-up to that of the NIST. The diameter of the integrating sphere is $1.65 \mathrm{~m}$. The spatial responsivity over the sphere surface is measured using high-intensity light-emitting diodes (LEDs) as beam light sources. For the measurement of the external flux, a commercial photometer (maintaining the illuminance unit) is used to determine the illuminance at the aperture plane. The luminous flux unit is maintained by the integrating-sphere facility and also by a group of standard lamps. The expanded uncertainty $(k=2)$ of the realization of the luminous flux unit is $0.78 \%$. The standard uncertainty components include the external luminous flux measurement $(0.21 \%)$, characterization of the sphere $(0.08 \%)$, determination of the sphere photometer responsivity and the internal luminous flux measurement $(0.19 \%)$, reproducibility of the measurements $(0.05 \%)$, and near-field absorption by the lamp socket and holder $(0.25 \%)$. The last uncertainty component is expected to be reduced significantly after implementation of a new lamp holder in the near future.

\section{Comparison measurements and results}

\subsection{Illuminance responsivity}

The comparison was carried out by measuring the illuminance responsivity (A/lx) of two transfer photometers (LMT P15 FOT, temperature-controlled type) at the HUT and the NIST. The transfer photometers were those used by the NIST at the 1997 key comparison CCPR-K3.b [12]. The photometers were calibrated at the NIST in June and August 2000, before and after transportation (by hand) to and from the HUT. The observed relative changes were less than $0.02 \%$. The maximum change was divided by $\sqrt{3}$ to calculate the standard uncertainty arising from transport $(0.01 \%)$ for a rectangular probability distribution. The HUT commercial photometer was also calibrated during the comparison as it was used to transfer the illuminance unit to the luminous flux facility.

At the NIST, the transfer photometers were measured twice on separate dates - before transportation to the HUT and after returning from the HUT. The measurements were made directly against the NIST reference photometers that maintain the NIST illuminance unit, using the calibration procedures described in [8]. The expanded uncertainty $(k=2)$ of the calibration of the transfer photometers with respect to the maintained NIST illuminance unit was estimated to be $0.07 \%$. The standard uncertainty components for this transfer measurement include photometer head distance alignment $(0.02 \%)$, effect of stray light $(0.02 \%)$, random noise including stability of the source $(0.02 \%)$, and transimpedance gain of the current-tovoltage converter $(0.01 \%)$. 
At the HUT, the measurements were performed on the optical bench using a luminous intensity standard lamp (Osram Wi41/G) operating at a colour temperature of $2856 \mathrm{~K}$. The light source and the photometers were aligned to the same optical axis using an alignment laser. Two stray-light screens between the light source and the photometers were used at distances of about $0.3 \mathrm{~m}$ and $1.5 \mathrm{~m}$ from the lamp.

The illuminance was measured at distances of $2.085 \mathrm{~m}, 2.585 \mathrm{~m}$ and $3.085 \mathrm{~m}$. The uncertainty in ensuring the same measurement positions for the NIST and HUT photometers was estimated to be $\pm 0.5 \mathrm{~mm}$, giving a standard uncertainty component of $0.03 \%$ at $2.085 \mathrm{~m}$. The photometer signals were amplified with different gains of the current-to-voltage converter, which causes an additional $0.01 \%$ standard uncertainty component. The illuminance was measured at the beginning and end of the comparison with the reference photometer at a distance of $3.085 \mathrm{~m}$. The time difference between these measurements was $1.5 \mathrm{~h}$. From the observed drift of the light source (relative value $-0.18 \%$ ) and the known time intervals between different measurements, a linear correction for the results was applied. The standard uncertainty of the drift correction was $0.02 \%$, arising from the \pm 5 min uncertainty of the measurement timing and $\pm 20 \%$ uncertainty in the linearity of the correction. Standard uncertainties of $0.01 \%$ from the stability of the transfer photometer, and $0.03 \%$ from the standard deviation of the results $(n=3)$ were also included. The overall expanded uncertainty of the calibration of the transfer photometer, with respect to the maintained HUT illuminance unit, was estimated to be $0.10 \%$ $(k=2)$.

Table 1 presents the results of the illuminance responsivity comparison. The average ratio of the measured illuminance responsivity values (HUT/NIST), was found to be 0.9992 with an expanded uncertainty $(k=2)$ of 0.0013 . From the combined standard uncertainties of the realizations of the units (NIST: $0.19 \%$, HUT: $0.09 \%$ ) and the uncertainty of the comparison, the expanded uncertainty $(k=2)$ of the agreement of the units between the HUT and the NIST is $0.47 \%$. The difference in the magnitude of the units maintained at the HUT and the NIST was found to be well below this expanded uncertainty. Table 2 shows the uncertainty budget for the comparison.

Table 1. Results of the illuminance responsivity comparison.

\begin{tabular}{lllll}
\hline \multirow{2}{*}{$\begin{array}{l}\text { Transfer } \\
\text { photometer }\end{array}$} & \multicolumn{3}{l}{ Measured illuminance responsivity/(A/lx) } & HUT/ \\
\cline { 2 - 5 } & $\begin{array}{l}\text { NIST } \\
\text { (before) }\end{array}$ & NIST (after) & HUT & NIST(ave.) \\
\hline$\# 1$ & $5.1317 \times 10^{-8}$ & $5.1313 \times 10^{-8}$ & $5.1279 \times 10^{-8}$ & 0.9993 \\
$\# 2$ & $5.1182 \times 10^{-8}$ & $5.1187 \times 10^{-8}$ & $5.1136 \times 10^{-8}$ & 0.9990 \\
Overall & & & & $\mathbf{0 . 9 9 9 2}$ \\
average & & & & \\
\hline
\end{tabular}

Table 2. Uncertainty budget for the illuminance responsivity comparison.

\begin{tabular}{ll}
\hline Source & $\begin{array}{l}100 \times \text { Relative } \\
\text { standard } \\
\text { uncertainty }\end{array}$ \\
\hline NIST calibration of transfer photometer (to NIST scale) 0.04 \\
HUT calibration of transfer photometer (to HUT scale) & 0.05 \\
Stability of transfer photometer during comparison & 0.01 \\
Combined standard uncertainty of comparison & 0.07 \\
Expanded uncertainty ( $k=2$ ) of comparison & \\
\hline Uncertainty of NIST illuminance unit [8] & 0.19 \\
Long-term stability of NIST illuminance unit [8] & 0.07 \\
Uncertainty of HUT illuminance unit [3] & 0.09 \\
Long-term stability of HUT illuminance unit [3] & 0.04 \\
Combined standard uncertainty of agreement & 0.23 \\
$\quad$ of units (\%) & \\
Expanded uncertainty ( $k=2$ ) of agreement & \\
$\quad$ of units $(\%)$ & $\mathbf{0 . 4 7}$ \\
\hline
\end{tabular}

\subsection{Luminous flux}

The comparison of the luminous flux units was carried out by calibrating four transfer lamps (Osram Wi41/Globe, prepared by the NIST) at both laboratories. The colour temperature of the lamps was $2750 \mathrm{~K}$ and stabilization time $10 \mathrm{~min}$. The ageing rate of these lamps was determined to be about $0.03 \% / \mathrm{h}$, which was included in the uncertainty budget for the stability of the transfer lamps.

The measurements at the NIST were carried out in June and August 2000, before and after transportation (by hand) to and from the HUT, in order to monitor any changes during transportation. The largest observed relative change of luminous flux was $0.02 \%$, which was included in the uncertainty budget. Each transfer lamp was measured twice (two burnings) each time. The relative expanded uncertainty $(k=2)$ of this calibration with respect to the 1997 NIST luminous flux unit is $0.14 \%$. The standard uncertainty components in this transfer measurement include lamp intensity distribution $(0.05 \%)$, self-absorption correction $(0.02 \%)$, spectral mismatch correction $(0.01 \%)$, near-field absorption $(0.02 \%)$ and lamp reproducibility $(n=4)(0.01 \%)$.

At the HUT, each lamp was operated and measured twice in the sphere and the measurement results were averaged. The maximum relative deviation of the two measurements was $0.03 \%$. The lamps were operated by a constant-current source. The lamp currents were monitored by a $100 \mathrm{~m} \Omega$ high-precision shunt resistor and a digital voltmeter. At the HUT, it was impossible to measure the voltage drop across the lamps with high accuracy as the socket of the lamp holder had a twopole configuration. The operating currents of the lamps were slightly different from those used at the NIST due to the limited resolution of the power supply. The maximum difference was $0.8 \mathrm{~mA}$ (NIST: $5.7959 \mathrm{~A}$; HUT: $5.7967 \mathrm{~A}$ ). The measured luminous flux values were corrected based on the sensitivity coefficient. The standard uncertainty component related to the correction 
for the difference in lamp current was estimated to be $0.03 \%$.

In the HUT set-up, only one power supply was used during the time of the comparison. The external source was therefore measured before and after the measurements of all the test lamps, and thus the responsivity of the sphere photometer (including lamp self-absorption) was calibrated with one of the four transfer lamps mounted in the sphere. As the variation of self-absorption of the lamps was later found to be not negligible, their self-absorption values were obtained from the values measured for the NIST sphere and transferred to the HUT sphere based on the selfabsorption sensitivity ratio. This ratio was obtained using a piece of black cardboard $(2.5 \mathrm{~cm}$ square $)$ sent from the NIST to the HUT as a transfer standard, which was placed inside each sphere and its absorption measured. The self-absorption correction in the HUT sphere was measured to be 3.41 times larger than that in the NIST sphere. This ratio was then used for responsivity calculations of the HUT sphere photometer for each lamp. The correction factors for the four lamps varied within $0.44 \%$. (For three lamps the selfabsorption corrections were equal within $\pm 0.04 \%$ ). The standard uncertainty assigned to the self-absorption correction was $0.02 \%$.

From the measured spatial response of the HUT sphere, the errors arising from the intensity distribution of the transfer lamps were estimated to be within $0.07 \%$, which is translated into a standard uncertainty component of $0.04 \%$. From the uncertainty components mentioned above, the relative expanded uncertainty $(k=2)$ of the HUT transfer measurement with respect to the HUT luminous flux unit is estimated to be $0.06 \%$.

Table 3 presents the results of the luminous flux comparison. The average ratio of the measured luminous flux values (HUT/NIST), was found to be 1.0006 with an expanded uncertainty $(k=2)$ of 0.0018 . From the combined standard uncertainties of the realizations of the units (1997 NIST: $0.31 \%$; HUT: $0.39 \%)$ and the uncertainty of the comparison, the expanded uncertainty $(k=2)$ of the agreement of the units between the HUT and the NIST is $1.01 \%$. The difference in the magnitude of the units maintained at the HUT and the NIST was found to be well below this expanded uncertainty. Table 4 shows the uncertainty budget for the comparison.

Table 3. Results of the luminous flux comparison.

\begin{tabular}{lllll}
\hline \multirow{2}{*}{$\begin{array}{l}\text { Transfer } \\
\text { lamp }\end{array}$} & \multicolumn{3}{c}{ Measured luminous flux/lm } & HUT/ \\
\cline { 2 - 5 } & NIST (before) & NIST (after) & HUT & NIST(ave.) \\
\hline TF8-3 & 2334.7 & 2334.7 & 2336.5 & 1.0008 \\
TF8-4 & 2307.7 & 2307.7 & 2307.6 & 1.0000 \\
TF8-5 & 2340.1 & 2340.5 & 2343.1 & 1.0012 \\
TF8-6 & 2361.4 & 2361.7 & 2362.6 & 1.0004 \\
Overall & & & & $\mathbf{1 . 0 0 0 6}$ \\
average & & & & \\
\hline
\end{tabular}

Table 4. Uncertainty budget for the luminous flux comparison.

\begin{tabular}{ll}
\hline Source & $\begin{array}{l}100 \times \text { Relative } \\
\text { standard } \\
\text { uncertainty }\end{array}$ \\
\hline $\begin{array}{l}\text { NIST calibration of transfer lamps } \\
\quad \text { to NIST 1997 scale) }\end{array}$ & 0.07 \\
HUT calibration of transfer lamps (to HUT scale) & 0.06 \\
$\begin{array}{l}\text { Stability of transfer lamps during comparison } \\
\text { Combined standard uncertainty of comparison }\end{array}$ & 0.01 \\
Expanded uncertainty $(k=2)$ of comparison & 0.09 \\
\hline Uncertainty of 1997 NIST unit on reference lamps & 0.31 \\
Uncertainty of HUT unit & 0.39 \\
Combined standard uncertainty of agreement of units & 0.51 \\
Expanded uncertainty $(k=2)$ of agreement of units & \\
\hline
\end{tabular}

\section{Conclusions}

The detector-based units of illuminance responsivity and luminous flux realized at the NIST and the HUT were compared. The results show that both units agree to within $0.1 \%$. The result of the illuminance responsivity comparison is consistent with that of key comparison CCPR-K3.b, where the ratio of the HUT and NIST values (HUT/NIST) was reported to be 0.9980 with an expanded uncertainty of 0.0018 , and the expanded uncertainty of the agreement of the units $0.72 \%$. The present measurement results indicate very good agreement for the illuminance and luminous flux units realized at the two laboratories using similar detector-based methods, and are encouraging towards the aim of achieving a $0.1 \%$ level of agreement in detector-based photometric units worldwide.

Note. Specific firms and trade names are identified in this paper to adequately describe the experimental procedure. Such identification does not imply recommendation or endorsement by the National Institute of Standards and Technology, nor does it imply that the materials or equipment identified are necessarily the best available for the purpose.

\section{References}

1. Cromer C. L., Eppeldauer G., Hardis J. E., Larason T. C., Ohno Y., Parr A. C., J. Res. Natl. Inst. Stand. Technol., 1996, 101, 109-132.

2. Ikonen E., Kärhä P., Lassila A., Manoochehri F., Fagerlund H., Liedquist L., Metrologia, 1995/96, 32, 689-692.

3. Toivanen P., Kärhä P., Manoochehri F., Ikonen E., Metrologia, 2000, 37, 131-140.

4. Ohno Y., J. IES, 1996, 25(1), 13-22.

5. Lahti K., Hovila J., Toivanen P., Vahala E., Tittonen I., Ikonen E., Metrologia, 2000, 37, 595-598.

6. Larason T. C., Bruce S. S., Parr A. C., Spectroradiometric Detector Measurements, Natl. Inst. Stand. Technol. Spec. Publ. 250-41, 1998.

7. Gentile T. R., Houston J. M., Hardis J. E., Cromer C. L., Parr A. C., Appl. Opt., 1996, 35(7), 1056-1068. 
8. Ohno Y., Photometric Calibrations, Natl. Inst. Stand. Technol. Spec. Publ. 250-37, 1997.

9. Ohno Y., Zong Y., Proc. 24th Session CIE, 1999, 1(1), 155-160.

10. Consultative Committee for Photometry and Radiometry, key comparisons CCPR-K3.a for luminous intensity and K4 for luminous flux, www.bipm.org/kcdb.

11. Ikonen E., Toivanen P., Lassila A., Metrologia, 1998, 35, 369-372.
12. Consultative Committee for Photometry and Radiometry, key comparison CCPR-K3.b for luminous responsivity, www.bipm.org/kcdb.

Received on 6 November 2001 and in revised form on 12 December 2001. 